\title{
An Approach of Drivetrain Degradation Assessment Applied in Wind Turbines
}

\author{
Menghang Zhang \\ Central Academe, Shanghai Electric Group Co., Ltd., China \\ siovanus@126.com
}

\begin{abstract}
Keywords: Feature extraction, Self-organizing map, Minimum quantization error, Contribution value, Faulty localization, Drivetrain degradation assessment.
\end{abstract}

\begin{abstract}
As wind energy is widely used nowadays, it is more and more important to predict wind turbine downtime and organize maintenance just in time. Two types of data systems have been widely adopted for monitoring wind turbine condition: supervisory control and data acquisition (SCADA) and condition monitoring system (CMS). In this paper, a systematic framework is designed to combine CMS and SCADA data and assess drivetrain degradation. Advanced feature extraction techniques are applied to calculate health indicators. A pattern recognition algorithm is used to model baseline and actual condition, Self-organizing Map (SOM) and minimum quantization error (MQE) method is selected to do the degradation assessment. Eventually, the contribution of each component is calculated to achieve component-level fault localization. The approach is validated on a $2 \mathrm{MW}$ wind turbine, where an incipient fault is detected before the wind turbine shuts down.
\end{abstract}

\section{Introduction}

In view of the increasingly energy crisis and environmental problems, wind energy has been highly concerned by various countries as a kind of green energy. According to the annual report of World Wind Energy Association (WWEA) in 2014, new installed capacity reached 23,196 MW in China, a 44.2 percent increase year-on-year. The whole installed capacity reached 114,609 MW, a 25.4 percent increase year-on-year, where 1.5MW and 2MW units occupied 87 percent.

In most of the available literatures, CMS and SCADA data are separately used. Moreover, most algorithms and techniques are developed and validated on test beds. So a degradation assessment framework is used to combine CMS data and SCADA variables to assess drivetrain degradation. The framework is eventually validated on a $2 \mathrm{MW}$ turbine drivetrain.

\section{Methodology}

The methodology selects both SCADA and CMS data of wind turbines, provides global degradation assessment and fault localization of the drivetrain.

The whole methodology and process are described as follows.

\subsection{SCADA Data Selection}

In SCADA systems, there are some variables that are related to the drivetrain. The fault in drivetrain can cause some difference in such variables. These variables are valuable for assessing the degradation condition of the drivetrain and its components. Examples of these variables include temperature of the rotor, gearbox and generator, as well as the gearbox oil pressure. For the reason that SCADA data is typically recorded more frequently than CMS data, SCADA records are selected only when CMS records exist at matching time interval.

\subsection{CMS Data Feature Extraction}

SCADA data can be directly used as condition indicators, but for CMS data, features must be extracted in order to obtain representative indicators. Signal processing techniques for vibration-based wind turbine monitoring has been developed, to extract features of drivetrain and components such as bearings, shafts, and gears.

Time Domain Features 
There are three commonly used features for vibration analysis: root mean square (RMS), kurtosis and crest factor. For a data vector $x_{i}, i=1,2,3 \ldots \mathrm{N}$, these features are defined as follows:

$$
\begin{aligned}
& \text { RMS }=\sqrt{\frac{\sum_{i=1}^{N} X_{i}^{2}}{N}}(1) \\
& \text { Kurtosis }=\frac{\sum_{i=1}^{N}\left(X_{i}-\bar{X}\right)^{4}}{N} /\left[\frac{\sum_{i=1}^{N}\left(X_{i}-\bar{X}\right)^{2}}{N}\right]^{2}
\end{aligned}
$$

Crest Factor $=\max \left(\left|X_{i}\right|\right) / R M S(3)$

Spectral Kurtosis Filtered Features

Spectral kurtosis filtering (SKF) is a technique to optimize the configuration of band-pass filter for noise reduction. Based on time-frequency analysis results, it adopts the kurtosis computation from the time domain analysis to seek the most impulsive frequency band.

As developed by Antoni (2006) for non-stationary signal analysis, the short-time Fourier transform (STFT) of the signal is first calculated as $\mathrm{H}(\mathrm{t}, \mathrm{f})$. For each frequency index decided by the STFT, the kurtosis of its amplitude over discrete time is calculated as Eq. (4):

$$
\operatorname{SK}_{X}(f)=\frac{\left\langle H^{4}(t, f)\right\rangle}{\left\langle H^{2}(t, f)\right\rangle^{2}}-2
$$

A statistical threshold $S_{\alpha}$ is computed (Antoni and Randall, 2006) to decide the significance of spectral kurtosis given level of significance $\alpha$ :

$$
\mathrm{S}_{\alpha}=u_{1-\alpha} \frac{2}{\sqrt{K}}(5)
$$

Where $u_{1-\alpha}$ is the quantile with significance level $\alpha$, and $\mathrm{K}$ is the number of time windows in STFT analysis.

An example of spectral kurtosis filtering is shown in Figure 1, where filtered data (plot in red) is more impulsive than raw data (plot in blue).
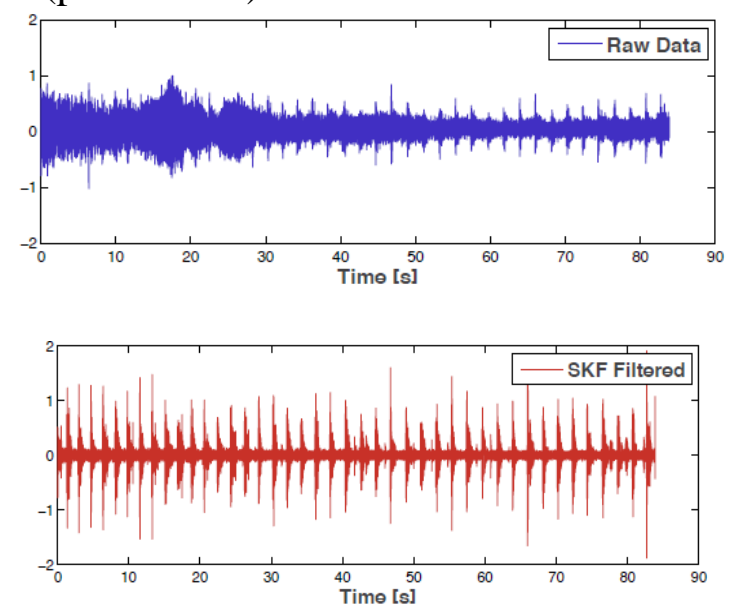

Envelope Features

Figure1. An example of SKF

Hilbert transform is used to obtain the envelope of a signal. After finding the envelope, time domain statistics can be calculated as condition indicators, such as RMS, kurtosis and crest factor (Eq.1, Eq.2 and Eq.3).

\subsection{Degradation Assessment}

In this paper, Self-organizing Maps (SOM) approach is used for degradation assessment. As an artificial neural network, SOM is able to automatically discover signal patterns and organize signals to create spatial separation between clusters.

Minimum quantization error (MQE) is the distance between baseline and actual sample, calculated as the Euclidean distance between an input vector and BMU, as shown in Eq. (6).

$\mathrm{MQE}=\left\|x-w_{B M U}\right\|(6)$

Now it is desirable to locate the fault at component level. The features can be grouped by drivetrain components based on their contextual information. For each component, its contribution to MQE value is calculated as Eq. (7), where $\mathrm{e}_{i}$ are features of the same component.

$$
\text { Contribution }=\frac{\sum e_{i}^{2}}{M Q E^{2}}
$$




\section{Case Study}

We validated the algorithm on a 2MW wind turbine. A split torque, three-stage planetary gearbox is used to connect the rotor and the generator on the drivetrain. Schematics of the drivetrain, as well as locations of vibration sensors, are shown in Figure 2.

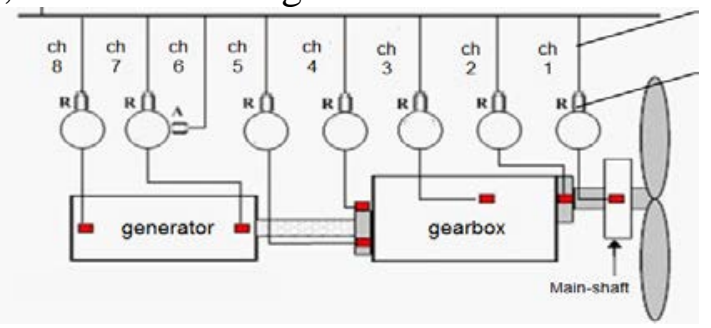

Figure2. Schematics of the drivetrain

In total, 22 CMS features and 4 SCADA variables are selected as condition indicators. For this case, SOM-MQE was used for calculation. And a Monte Carlo based statistic method is adopted to generate a threshold. Eventually the trend of MQE and corresponding SCADA variables are shown in Figure 3.
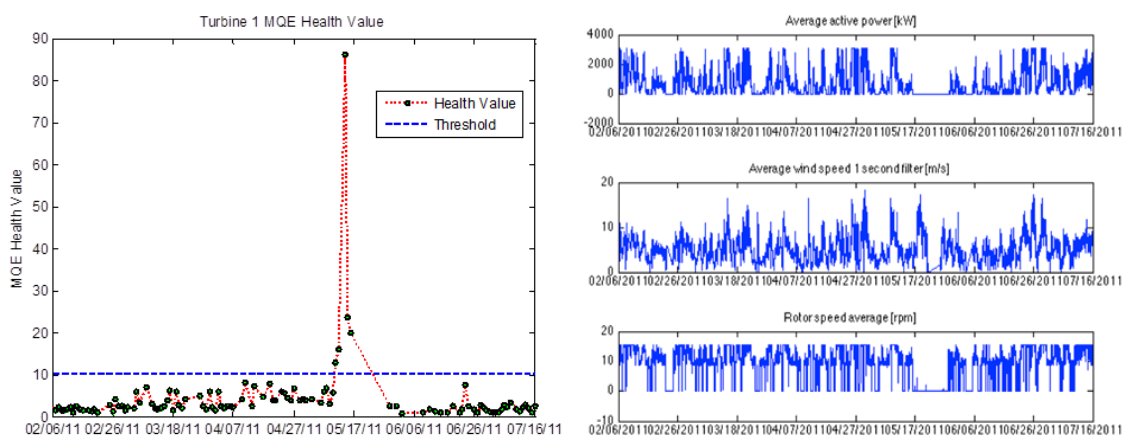

Figure3. The MQE value and corresponding SCADA data
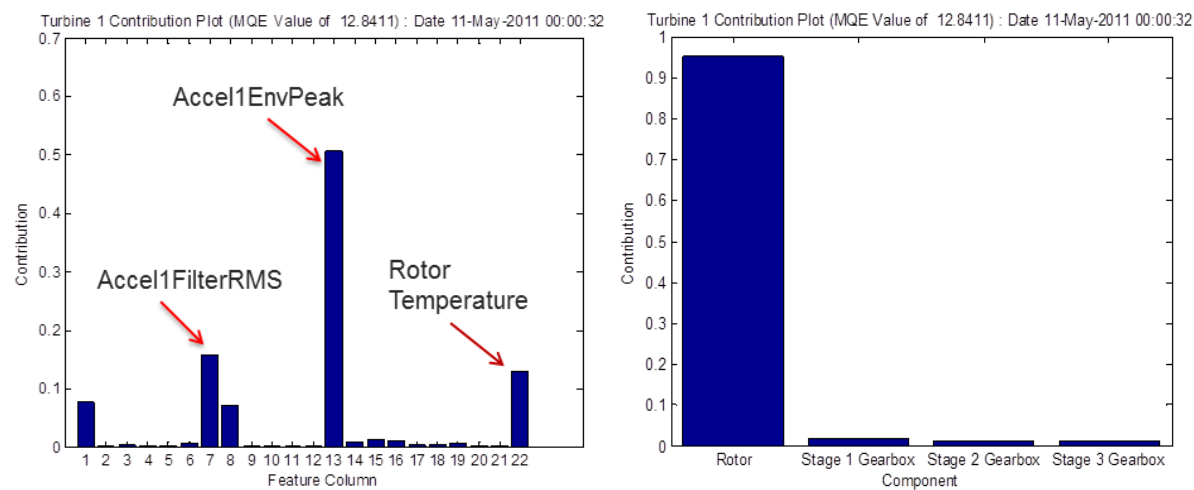

Figure4. The contribution of each feature and component

\section{Discussion}

In this paper, an integrated methodology of degradation assessment and fault localization for wind turbine drivetrain components is presented. The result of the methodology is achieved by combining input from SCADA system and CMS, and validated with a planetary gearbox system of 2MW wind turbine.

Besides using SCADA variables, feature extraction methods are also used to extract condition indicators from CMS data. The inputs include time domain features, spectral kurtosis filtering and envelope analysis. A Self-organizing Map and minimum quantization error approach is adopted to assess the degradation of drivetrain, and contribution calculation is used to decide the location of defect on the drivetrain. In the case study, an incipient defect is detected and located. 


\section{References}

[1] Antoni, J. (2006). The spectral kurtosis: a useful tool for non-stationary signals. Mechanical Systems and Signal Processing, 20 (2), pp. 282-307.

[2] McMillan, D., \& Ault, G. W. (2007). Quantification of condition monitoring benefit for offshore wind turbines. Wind Engineering, 31 (4), pp. 267-285.

[3] Bechhoefer, E., He, D., \& Dempsey, P. (2011). Gear health threshold setting based on a probability of false alarm. Annual Conference of the Prognostics and Health Management Society, September 25 - 20, Montreal, Canada. 